\title{
SEMISIMPLICITY OF GROUP ALGEBRAS OF VECTOR-VALUED FUNCTIONS
}

\author{
D. Z. SPICER
}

1. Introduction. Let $G$ be a compact group with normalized Haar measure, $1 \leqq p<\infty$, and $A$ be a Banach algebra. Define $B^{p}(G, A)$ to be the set of equivalence classes, modulo null functions, of all functions $f: G \rightarrow A$ such that $\int_{G}\|f(x)\|{ }^{p} d x<\infty$. Similarly, define $C(G, A)$ to be the set of all continuous functions from $G$ to $A$. These sets form Banach algebras under the usual operations and convolution multiplication. These Banach algebras are studied in detail in [5].

We say that a Banach algebra is strongly semisimple if its strong radical, i.e. the intersection of all modular maximal ideals, vanishes. In [5] it is determined that for any compact group $G$ and $1 \leqq p$ $<\infty, B^{p}(G, A)$ (or $C(G, A)$ ) is strongly semisimple if and only if $A$ is strongly semisimple.

For noncommutative algebras there are other types of "semisimplicity" that are of interest. In particular, we say that a Banach algebra is semisimple if its Jacobson radical vanishes. It is readily seen (cf. [5]) that a necessary condition for any $B^{p}(G, A)$ (or $C(G, A)$ ) to be semisimple is that $A$ be semisimple. Further in [5] it is conjectured, in analogy to the strongly semisimple case, that this condition is also sufficient. Here we show that this conjecture is correct.

2. Theorem. If $G$ is a compact group, $1 \leqq p<\infty$, and $A$ is a semisimple Banach algebra, then $B^{p}(G, A)$ (or $\left.C(G, A)\right)$ is also semisimple.

Proof. Since $B^{p}(G, A)$ (or $C(G, A)$ ) is isomorphic to an ideal of $B^{1}(G, A)$, it is sufficient to prove the theorem for $B^{1}(G, A)$. In [5] it is noted, following Grothendieck, that $B^{1}(G, A)$ is isomorphic to $L^{1}(G) \otimes_{\gamma} A$, where $\gamma$ is the "greatest cross-norm". This fact allows us to use the known structure of $L^{1}(G)$ to reduce the scope of the problem to a more manageable form. (The reader is referred to [5] for the notation and properties of topological tensor products used in this paper.)

Lemma 1. Let $X_{1}$ be a Banach space of dimension $n<\infty$, and let $X_{2}$ be any Banach space, then the algebraic tensor product of $X_{1}$ and $X_{2}$, $X_{1} \otimes X_{2}$, is complete under the $\gamma$-norm.

Presented to the Society, January 24, 1967 under the title Group algebras of zectorvalued functions; received by the editors December 6, 1966. 
Proof. We first note that $X_{1} \otimes X_{2}$ is isomorphic to the direct sum of $X_{2}$ with itself $n$ times. In fact, if $\left\{e_{i}: 1 \leqq i \leqq n\right\}$ is a basis for $X_{1}$ with $\left\|e_{i}\right\|=1$ for all $i$, then every tensor $T \in X_{1} \otimes X_{2}$ has one and only one representative of the form $\sum_{i=1}^{n} e_{i} \otimes y_{1}$. Letting then $\phi(T)$ $=\left(y_{1}, \cdots, y_{n}\right)$, it is seen that $\phi$ is a well-defined isomorphism.

We next note that the above direct sum is complete under the norm

$$
\left\|\left(y_{1}, \cdots, y_{n}\right)\right\|=\sum_{i=1}^{n}\left\|y_{i}\right\| .
$$

Further, for any $T \in X_{1} \otimes X_{2}$ with representative $\sum_{i=1}^{n} e_{i} \otimes y_{i}$, we see that

$$
\|T\|_{\gamma} \leqq \sum_{i=1}^{n}\left\|e_{i}\right\|\left\|y_{i}\right\|=\sum_{i=1}^{n}\left\|y_{i}\right\|=\|\phi(T)\|
$$

We get an opposing inequality by noting first that by the uniqueness of the norm topology in finite-dimensional spaces, there is a constant $c$ such that $c\|x\| \geqq\|x\|_{1}$ for every $x \in X_{1}$, where $\|x\|_{1}$ is the $l_{1}$-norm of $x$ (i.e. if $\left.x=\sum_{i=1}^{n} \lambda_{i} e_{i},\|x\|_{1}=\sum_{i=1}^{n}\left|\lambda_{i}\right|\right)$. Now again take $T \in X_{1} \otimes X_{2}$, and let $\sum_{j=1}^{m} x_{j} \otimes y_{j}$ be an arbitrary representative of $T$. If for each $1 \leqq j \leqq m, x_{j}=\sum_{i=1}^{n} \lambda_{i j} e_{i}$, then

$$
\sum_{i=1}^{n} e_{i} \otimes\left(\sum_{j=1}^{m} \lambda_{i j} y_{j}\right)
$$

is also a representative of $T$. Therefore,

$$
\begin{aligned}
\|\phi(T)\| & =\sum_{i=1}^{n}\left\|\sum_{j=1}^{m} \lambda_{i j} y_{j}\right\| \\
& \leqq \sum_{i=1}^{n} \sum_{j=1}^{m}\left|\lambda_{i j}\right|\left\|y_{j}\right\| \\
& \leqq c \sum_{j=1}^{m}\left\|x_{j}\right\|\left\|y_{j}\right\| .
\end{aligned}
$$

Since this inequality holds for every $\sum_{j=1}^{m} x_{j} \otimes y_{j}$ representing $T$, $\|\phi(T)\| \leqq c\|T\|_{\gamma}$.

These two inequalities and the fact that the direct sum is complete under the indicated norm yield the desired conclusion. Note that $\phi$ is also seen to be a homeomorphism.

Lemma 1 has the effect of reducing our problem to a purely algebraic one that is readily solved.

We now note (cf. [2]) that $L^{1}(G)$ is a semisimple dual Banach 
algebra, and as such is the topological direct sum of its minimal closed ideals. Each of these minimal closed ideals in turn is continuously isomorphic to the algebra of all linear operators on a finite-dimensional Banach space.

Lemma 2. If $M$ is a minimal closed ideal of $L^{1}(G)$ and $A$ is a primitive Banach algebra, then $M \otimes_{\gamma} A$ is also a primitive Banach algebra.

Proof. By the above remark, $M$ is isomorphic to the algebra $\mathfrak{L}\left(X_{1}\right)$ of all linear operators on a finite-dimensional space $X_{1}$. Further since $A$ is primitive, $A$ is isomorphic to a strictly dense subalgebra $B \subseteq \mathfrak{L}\left(X_{2}\right)$ for some linear space $X_{2}$. By means of these identifications, $M \otimes A$ can be isomorphically embedded in $\mathscr{L}\left(X_{1} \otimes X_{2}\right)$ in a natural way. This representation is also irreducible as can be easily verified directly or as a consequence of $[1$, p. 114]. Thus by Lemma 1 , $M \otimes_{\gamma} A$ is primitive.

Lemma 3. If $M$ is a minimal closed ideal of $L^{1}(G)$, and $A$ is a semisimple Banach algebra, then $M \otimes_{\gamma} A$ is also a semisimple Banach algebra.

Proof. Since $A$ is semisimple, $A$ is isomorphic to a subdirect sum $\sum_{\lambda}^{s} A_{\lambda}$ of primitive Banach algebras $A_{\lambda}(\lambda \in \Lambda)$. Since $M$ is finitedimensional, Lemma 1 is again applicable. Define then

$$
\mu: M \otimes_{\gamma} A \rightarrow \sum_{\lambda}\left(M \otimes_{\gamma} A_{\lambda}\right)
$$

(where the right-hand side denotes the complete direct sum) in the following manner. Let $\left\{f_{i} \mid 1 \leqq i \leqq m\right\}$ be a basis for $M$ with $\left\|f_{i}\right\|=1$ for all $i$, and let $\sum_{i=1}^{m} f_{i} \otimes a_{i}$ represent an arbitrary tensor $T \in M \otimes_{\gamma} A$. If for each $1 \leqq i \leqq m,\left\{a_{i}(\lambda): \lambda \in \Lambda\right\} \in \sum_{\lambda}^{s} A_{\lambda}$ corresponds to $a_{i}$, let $\mu(T)=\left\{T_{\lambda}: \lambda \in \Lambda\right\}$ where for each $\lambda \in \Lambda, T_{\lambda}$ is the element of $M \otimes_{\gamma} A_{\lambda}$ with representative $\sum_{i=1}^{m} f_{i} \otimes a_{i}(\lambda)$. $\mu$ is easily seen to be a welldefined homomorphism into the complete direct sum. Further since all of the tensors above have one and only one representative of the above form, $\mu$ is seen to be one-to-one. Finally since $A$ is isomorphic to a subdirect sum, the image of $\mu$ is also a subdirect sum.

Thus we have shown that $M \otimes_{\gamma} A$ is isomorphic to a subdirect sum of primitive algebras, and as a result $M \otimes_{\gamma} A$ is semisimple.

Only one more remark need be made before we can proceed to prove the theorem. We note that in general the $\gamma$-norm is not of local character (cf. [5]). This means that if $M$ is a subalgebra of $L^{1}(G)$ and $N$ is a subalgebra of $A$, it is certainly true that $M \otimes N \subseteq L^{1}(G) \otimes A$, but it may not be true that $M \otimes_{\gamma} N \subseteq L^{1}(G) \otimes_{\gamma} A$, i.e. the $\gamma$-norm on 
$L^{1}(G) \otimes A$ is not an extension of the $\gamma$-norm on $M \otimes N$. However, in the present situation, where $M$ is a minimal closed ideal of $L^{1}(G)$, and thus finite-dimensional, and $N=A$, it is clear from Lemma 1 that the above problem does not arise (see also $[4$, p. 56]). It is further seen in this case that $M \otimes_{\gamma} A$ is a closed ideal of $L^{1}(G) \otimes_{\gamma} A$ since the $\gamma$-norm is compatible with multiplication (cf. [5]). It should be remarked here that it is crucial to our argument that $M \otimes_{\gamma} A$ is in fact an ideal. Since it is not in general true that the algebraic tensor product of two ideals is an ideal of the topological tensor product, we have been quite careful to indicate the fact that we are dealing with an ideal of $L^{1}(G) \otimes_{\gamma} A$ by the maintenance of a somewhat artificial distinction between $M \otimes A$ and $M \otimes_{\gamma} A$.

Now let $\Re$ be the radical of $L^{1}(G) \otimes_{\gamma} A=B^{1}(G, A)$ and assume $\Re \neq(0)$. If $\Re \cap\left(M \otimes_{\gamma} A\right)=(0)$ for every minimal closed ideal $M$ of $L^{1}(G)$, then $\Re \cdot\left(M \otimes_{\gamma} A\right)=(0)$ also for every such $M$. Since, as previously noted, $L^{1}(G)$ is the topological direct sum of its minimal closed ideals, it then is easily seen that $\Re \cdot\left(L^{1}(G) \otimes_{\gamma} A\right)=(0)$ also. However, this is impossible since $A$ being semisimple implies that only zero annihilates $B^{1}(G, A)$ under left multiplication. In fact, if $f \in B^{1}(G, A)$ is such that $f * g=0$ for all $g \in B^{1}(G, A)$, then for every $a \in A$ and measurable set $E \subseteq G, f *\left(a \chi_{E^{-1}}\right)=0$ (where $\chi_{E^{-1}}$ is the characteristic function of the set $\left.E^{-1}\right)$. But also

$$
\left(f *\left(a \chi_{E^{-1}}\right)\right)(x)=\left(\int_{x E} f(y) d y\right) a .
$$

Therefore, $\left(\int_{x E} f(y) d y\right) a=0$ for all $a \in A$, for all measurable sets $E \subseteq G$, and almost every $x \in G$. Since $A$ is semisimple, the left regular representation of $A$ on itself is faithful, and as a result $\int_{x E} f(y) d y=0$ for all measurable $E$ and almost every $x$. Taking such an $x_{0}$ and considering measurable sets of the form $x_{0}^{-1} E$, we see that $\int_{E} f(y) d y=0$ for all measurable sets $E$. This is possible only if $f=0$.

As a result of the above argument $\Re \cap\left(M \otimes_{\gamma} A\right) \neq(0)$ for some minimal closed ideal $M$. But on the other hand, since $M \otimes_{\gamma} A$ is an ideal of $L^{1}(G) \otimes_{\gamma} A, \Re \cap\left(M \otimes_{\gamma} A\right)$ is the radical of $M \otimes_{\gamma} A$, and thus must be (0) by Lemma 3 . Therefore, the initial assumption that $\Re \neq(0)$ must be false.

3. Remark. While this manuscript was in preparation, J. McCoy communicated that he had independently answered the conjecture stated in [5] (see [3]). His approach is quite different. First of all, he concentrates on $C(G, A)$ instead of on $B^{1}(G, A)$. This is indeed sufficient however (see [5]). Secondly instead of reducing the prob- 
lem as we have done, he attacks it directly and uses properties of irreducible unitary representations of $G$ in order to get a hold on enough irreducible representations of $C(G, A)$ to show that the intersection of their kernels, and thus also the radical, vanishes.

\section{BIBLIOGRAPHY}

1. N. Jacobson, Structure of rings, Amer. Math. Soc. Colloq. Publ., Vol. 37, Amer. Math. Soc., Providence, R. I., 1964.

2. I. Kaplansky, Dual rings, Ann. of Math. 49 (1948), 689-701.

3. J. McCoy, Generalized almost periodic functions, Thesis, Univ. of Oregon, Eugene, 1966.

4. R. Schatten, A theory of cross-spaces, Ann. of Math. Studies, No. 26, Princeton Univ. Press, Princeton, N. J., 1950.

5. D. Spicer, Group algebras of vector-valued functions, Pacific J. Math. (to appear).

University of California, Los Angeles 